\title{
Two cases of Van Buchem's disease
}

\author{
JM DIXON, ${ }^{*}$ RE CULL, $\dagger$ P GAMBLE. $\ddagger$ \\ From the Departments of Clinical Surgery, ${ }^{*}$ Medical Neurology, $\dagger$ and Radiology, $\ddagger$ Royal Infirmary, \\ Edinburgh, Scotland
}

SUMMARY A brother and sister suffering from hyperostosis corticalis generalisata familiaris (van Buchem's disease) are described. Both presented in early adult life with signs and symptoms of raised intracranial pressure and underwent partial craniectomy. Following surgery normal intellectual function was maintained and both survived to old age. In later life one showed cerebellar deficit due to bony encroachment of the posterior cranial fossa, while the other had a spastic paraparesis due to spinal cord compression. Several other siblings were affected by this disease which appears to be transmitted as an autosomal recessive gene.

In 1955 van Buchem, Hadders and Ubbens described a generalised skeletal disease in a twin brother and sister." They designated the disease "hyperostosis corticalis generalisata familiaris" and described the diagnostic features of gross thickening of the skull, mandible, clavicles, ribs and diaphyses of long bones. The neurological features in these cases were headache, visual failure, mental impairment, deafness and in later cases facial palsy. ${ }^{2}$ Other reports have confirmed these features. ${ }^{3-8}$ We report a brother and sister suffering from van Buchem's disease both presenting more than 20 years before this condition was first described, both of whom were successfully treated by partial craniectomy in early adult life for raised intracranial pressure. Normal intellectual function was maintained throughout life and each survived more than 50 years after surgery. The case reports of both are presented and the disease and its management discussed.

\section{Case reports}

Case 1 Miss $M M$ This 68-year-old spinster had suffered from deafness since her childhood and had been aware of bilateral facial weakness since her teens. At the age of 18 yr (1929) she had presented with headache, nausea and vomiting and on examination signs of raised intracranial pressure were present. Professor Norman Dott removed a large part of the right parietal bone, radiographs at that time having shown grossly thickened bone, which was thought to be compressing the cerebral hemispheres. Following operation she remained well until the age of 63 , when she

Address for reprint requests: Dr JM Dixon, University Department of Clinical Surgery, Royal Infirmary, Edinburgh EH3 9YW, Scotland, UK.

Received 29 April 1982. Accepted 6 June 1982 developed progressive unsteadiness of gait. She gave details of three of her siblings who were similarly affected (fig 1). Examination showed her skull to be irregularly thickened with coarse thickening of the mandible, clavicles, fingers and tibiae. Her gait was ataxic and broad based. Higher mental function was normal for her age. Visual fields were intact; ophthalmoscopic nystagmus was present with fine rotatory nystagmus on both right and left gaze. There was bilateral facial weakness. Tuning fork tests suggested bilateral conductive deafness. An intention tremor was present in both arms and legs and tendon reflexes were pathologically brisk in both arms and legs but plantar responses were flexor. Investigations showed normal chemistry apart from a raised alkaline phosphatase of 286 $\mathrm{u} / \mathrm{l}$ (normal 40-100 u/l), of bone origin. Serum parathyroid hormone and calcitonin were normal. Audiometry showed bilateral deafness principally conductive in type with sensineural deafness at higher frequencies. A skull radiograph (fig 2 ) showed marked thickening of the cranial vault particularly of the skull base and petrous bones. A localised exostosis was noted in the occipital region on the left. A bony defect was also present in the right parietal region, from the previous decompressive surgery. A chest

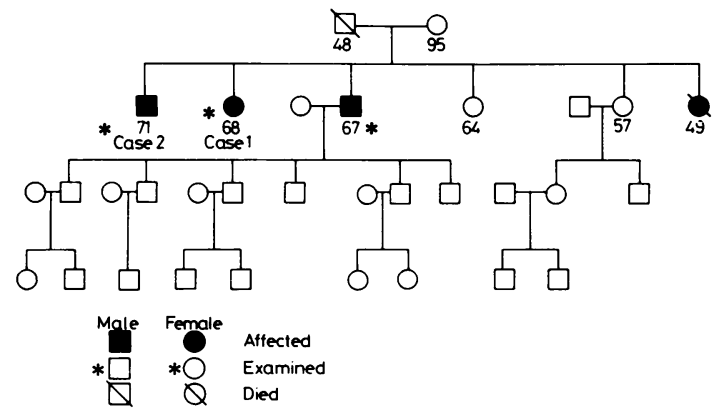

Fig 1 Family tree. 


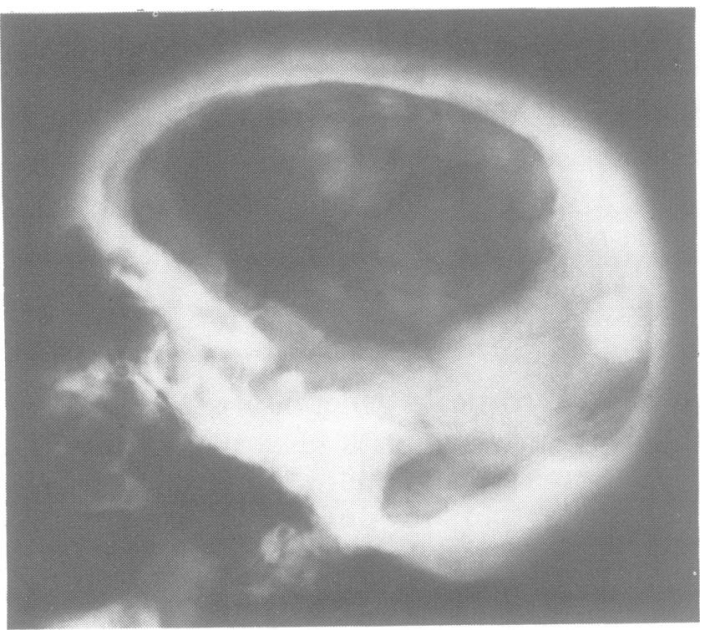

Fig 2 Lateral radiograph of the skull of the first patient showing very marked, rather nodular thickening of the cranial vault and particularly the skull bases with marked sclerosis of the petrous bones. A localised exostosis is present in the left occipital region. The right parietal defect is from the previous craniotomy.

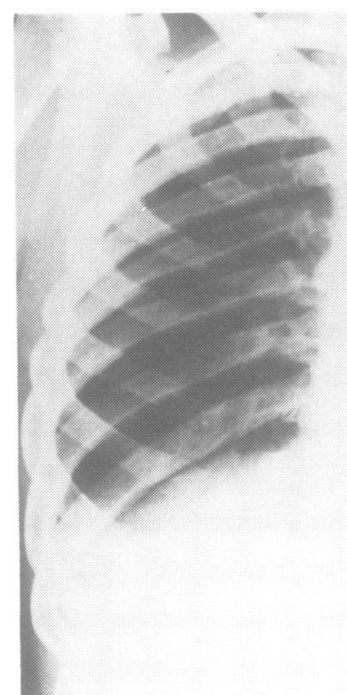

Fig 3 Chest radiograph of the first patient showing symmetrical cortical thickening of all the ribs and the clavicles. The heart and lung fields appear normal.

radiograph (fig 3) showed symmetrical thickening of the cortex of the ribs and clavicles. Radiographs of the hands (fig 4) showed considerable cortical thickening and sclerosis of all the metacarpals and phalanges, particularly affecting the diaphyses, although the bases of the proximal phalanges and metacarpals were also involved. A CT scan (fig 5) confirmed the considerable thickening of the vault, particularly at its base, where there was considerable

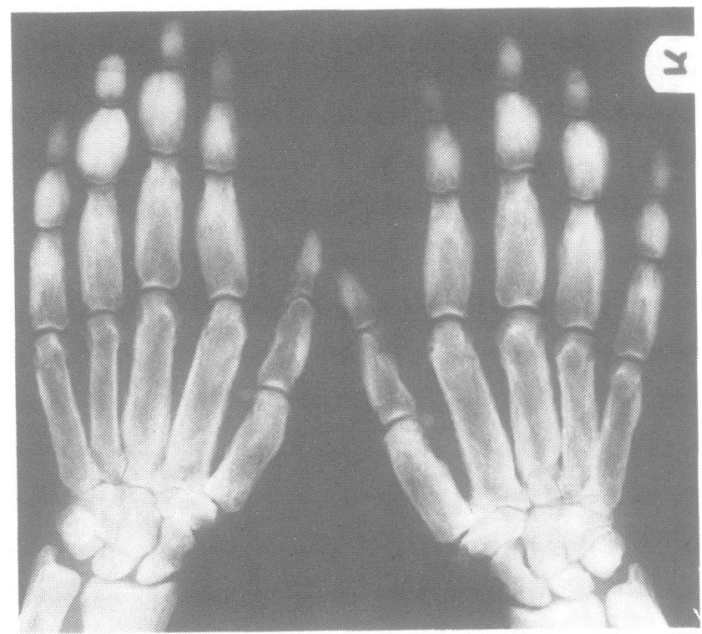

Fig 4 Hand radiographs of the first patient showing the marked, predominately diaphyseal cortical thickening of the metacarpals and phalanges. This is noted to extend to the bases of the metacarpals.

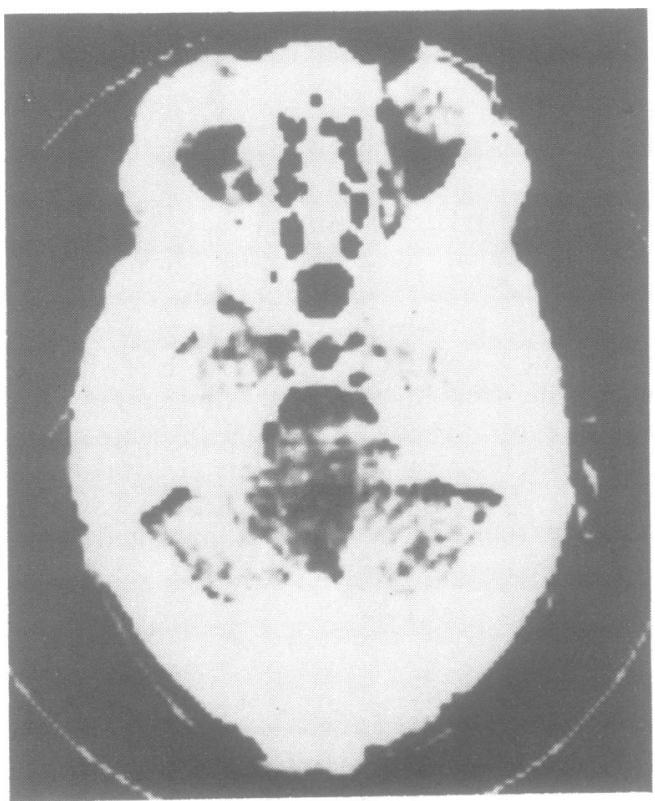

Fig 5 CT scan of case 1 showing very marked thickening of the skull of the posterior fossa with encroachment on the cerebellum.

encroachment of the posterior cranial fossa. The right parietal defect was seen to be partially covered by a thin layer of bone, which had grown since excision some 50 years earlier. The ventricular system appeared normal. It was decided that further surgical decompression in this patient was not possible and no specific treatment was instituted. During a long stay in hospital she became jaundiced. 


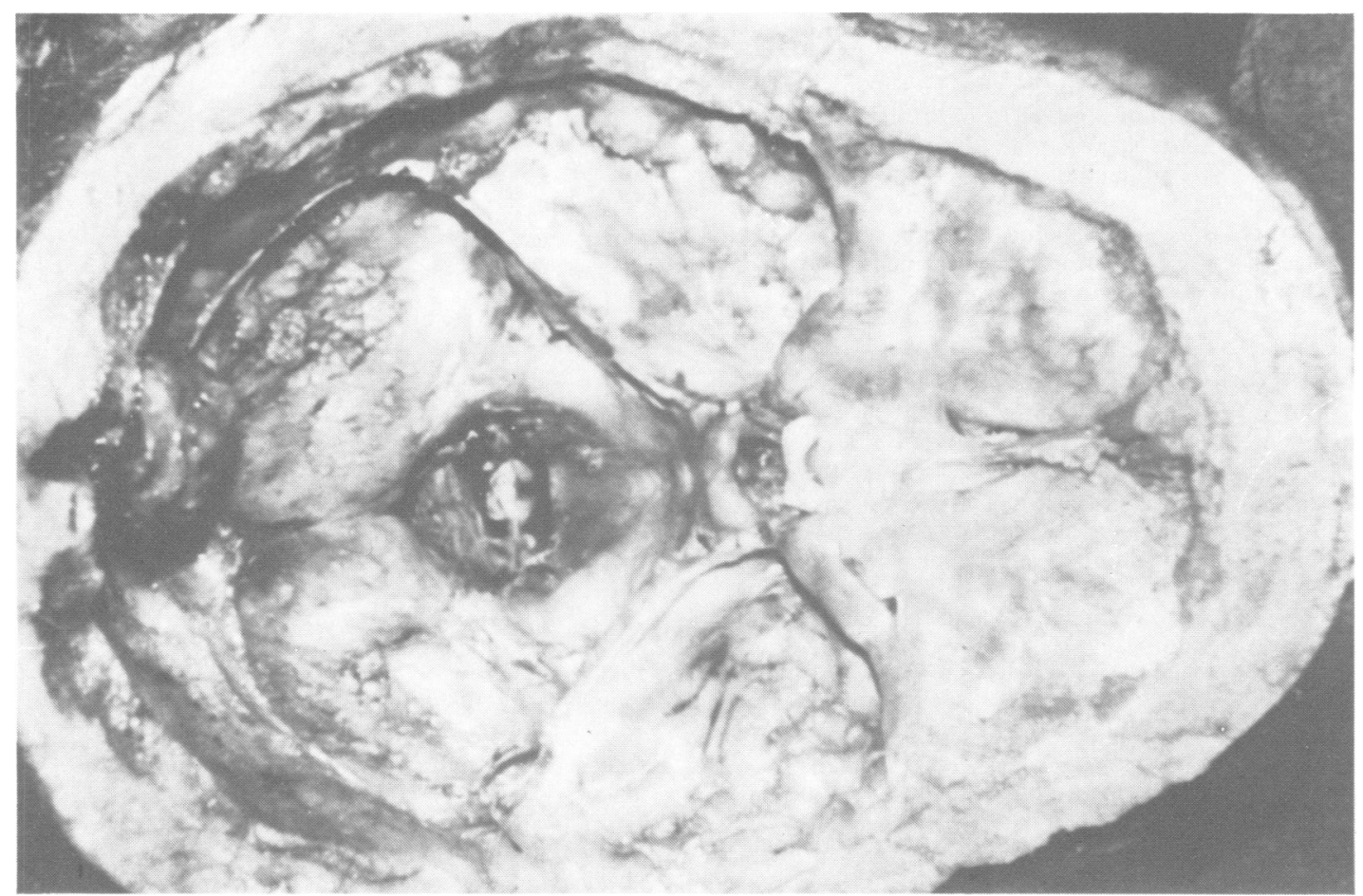

Fig 6 Case 1-skull at necropsy showing the marked nodular thickening of the cranial vault with the localised exostosis in the left occipital region. The skull thickness was $2 \cdot 2 \mathrm{~cm}$.
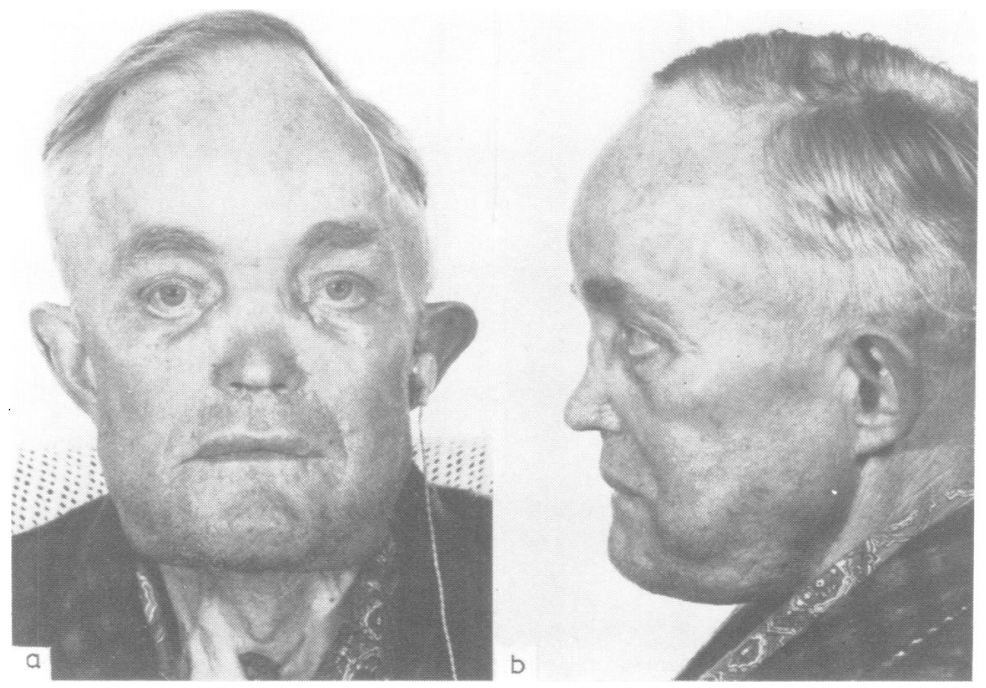

Fig 7a, b Mr AM. Facial appearance.

Investigation revealed the presence of a carcinoma of the transverse colon with multiple hepatic metastases. Her condition rapidly deteriorated and she died. A necropsy was performed and confirmed the cause of death as carcinoma of the colon with spread to regional lymph nodes and liver. The skull measured $2 \cdot 2 \mathrm{~cm}$ in thickness and consisted entirely of dense bone. Multiple localised exostoses were present over the external and internal surfaces of the skull vault (fig 6). Cortical thickening was present in all ribs, both clavicles and all long bones. 
Microscopy showed the increase in thickness of the bones to consist of normal lamellar bone. The brain showed deformity corresponding to the marked irregularity of the internal surface of the skull and a right cerebral convexity hernia was present. No secondary deposits of tumour were identified. The cerebellum similarly showed irregularity in contour but no other macroscopic or microscopic abnormality was present. In particular there was no evidence of cerebellar atrophy, which might have been a non-metastatic complication of the colon carcinoma.

Case $2 \mathrm{Mr}$ AM This 70-year-old retired church minister, the brother of the patient in case 1 developed weakness of first one side and later both sides of his face during his schooldays. At the age of $24 \mathrm{yr}$ (1932) he developed an unsteady gait, poor vision, headache, nausea and vomiting and had signs of raised intracranial pressure. He underwent bilateral craniectomies with removal of flaps of both parietal bones. His condition improved sufficiently for him to attend university and lead an active life. However, at age 30 he became deaf and at age 58 he developed progressive weakness and coldness in his left leg and by the age of 65 he was unable to walk. Recent symptoms included increasing urgency and frequency of micturition not helped by prostatectomy. On examination his facial appearance was abnormal (fig 7). The skull was irregularly thickened as was his mandible and maxilla; the digits, clavicles and tibae all showed fusiform thickening. Higher mental function was normal for his age. He was dysarthric owing to bilateral facial weakness and corneal reflexes were absent. Visual fields were intact, but there was pallor of optic discs. Tuning fork tests suggested bilateral conductive deafness. Muscle power, co-ordination and sensation were normal in the upper limbs. Tone was increased in a spastic fashion in the legs, particularly the left, where power was markedly reduced in all muscle groups (MRC grade 3). Sensation

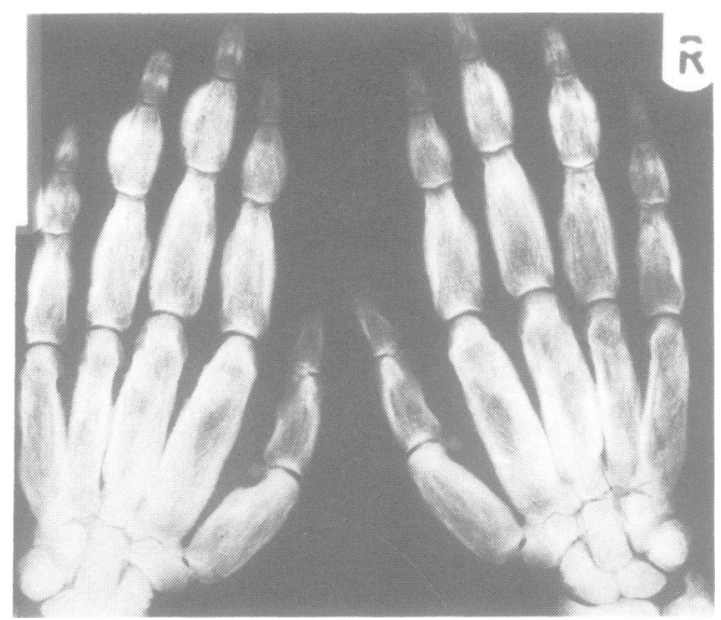

Fig 8 Hands of the second patient showing a similar appearance to those in Case 1.

to pin prick was impaired below L2 dermatome and proprioception was impaired in the left foot. Tendon reflexes were pathologically brisk in all four limbs particularly in the left leg. Hoffman's reflex was present bilaterally and the left plantar was extensor, the right flexor. Investigations showed the only serological abnormality to be a raised alkaline phosphatase of $680 \mathrm{u} / 1$ (normal 40-100 $\mathrm{u} / \mathrm{l}$ ). Radiographs of the skull showed previous bilateral craniectomy and generalised cortical thickening, as in the previous case but even more marked. In the long bones and hands there was hyperostosis and sclerosis particularly involving the diaphysis with relative sparing of the bone ends (fig 8). Spine radiographs showed similar generalised

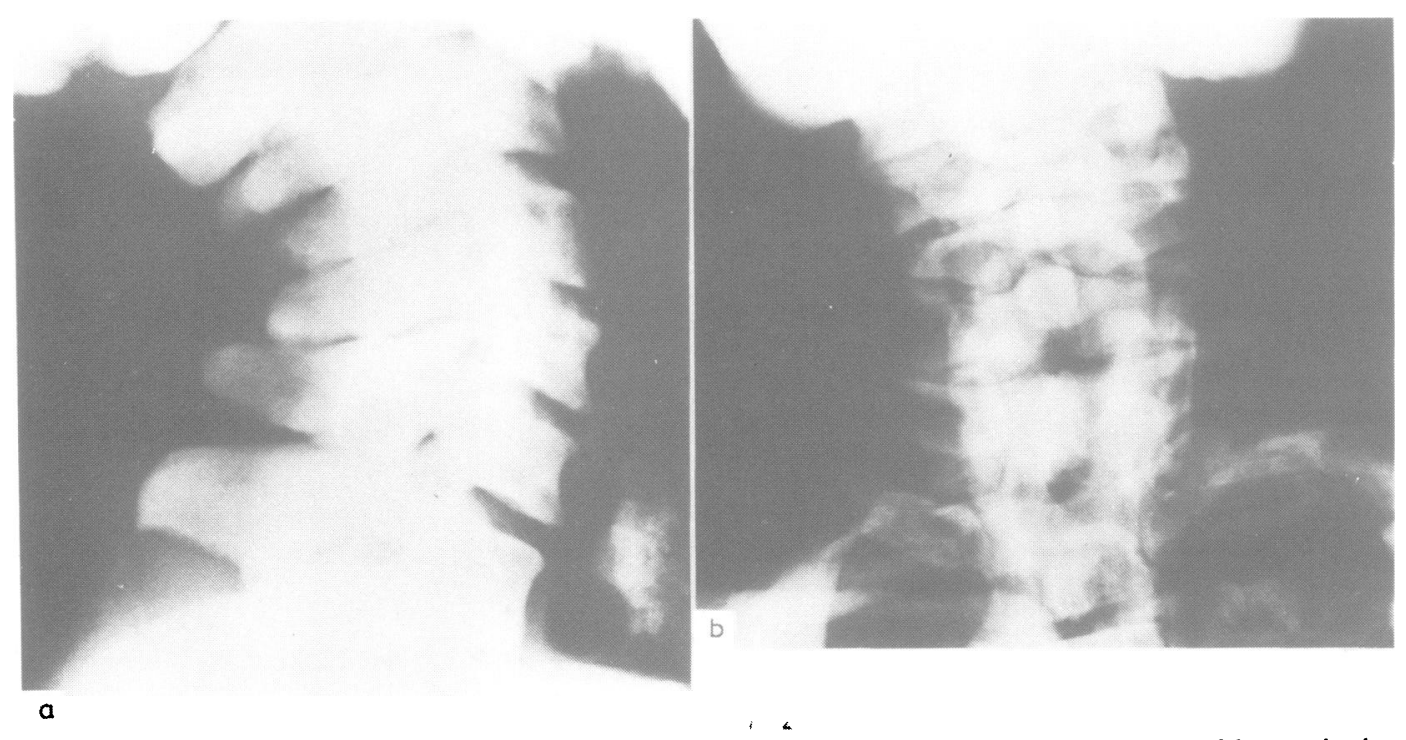

Fig 9 (a) Lateral and (b) AP radiographs of the cervical spine of the second patient, showing sclerosis of the vertebral bodies and posterior arches. The calcified laryngeal cartilage is also noted to be sclerotic. 
thickening and bony sclerosis, particularly involving the cervical spine (fig 9). The posterior vertebral arches were particularly affected. It was also noted the hyoid bone and the thyroid and cricoid cartilages were also increased in density.

\section{Discussion}

Van Buchem's disease may be differentiated from other forms of widespread bony sclerosis, such as osteopetrosis (Albers-Schonberg's disease), myelosclerosis and progressive diaphyseal dysplasia (Engleman Camurati disease), in several ways, and an elegant discussion on this differentiation has been presented elsewhere. ${ }^{129}$ Van Buchem's disease causes bony cortical thickening along the shafts of long bones, clavicles, ribs and to a major degree the skull bones and mandible. In contrast, the pelvis and metaphyses show less marked changes. Although osteopetrosis may affect the skull ${ }^{1011}$ with cranial nerve palsies due to encroachment on neural exit foramina, this is uncommon and in progressive diaphyseal dysplasia, skull changes are rarely seen. Both these diseases show sparing of the mandible which is markedly involved in van Buchem's disease. Van Buchem's disease is not usually associated with anaemia, a feature of osteopetrosis and myelosclerosis. A further distinction may be made on the basis of the elevation of serum alkaline phosphatase of bone origin seen in most cases of van Buchem's disease and present in the two cases reported here. No elevation in alkaline phosphatase is seen in osteopetrosis, where the main defect appears to be failure of bony resorption. ${ }^{12}$ Increased bone deposition occurs in van Buchem's disease, ${ }^{2}$ while hormones controlling bone resorption-calcitonin and parathyroid hormone-were present in normal concentrations in our first patient.

Most patients with van Buchem's disease develop facial palsy on one or both sides; presumably because of thickening of the walls of the facial canal. The deafness, which also occurs frequently, has been thought to be due to encroachment on the acoustic nerve in the internal auditory meatus. ${ }^{2}$ Both the present patients however, had principally conductive deafness, both on examination and on audiometry. This suggests that the bony sclerosis also affects the auditory ossiscles. One previous patient has also reported to have a combination of conductive and perceptive deafness. ${ }^{5}$

Symptoms of raised intracranial pressure were present in both our patients and are not uncommon in this disorder, ${ }^{15}$ although no record exists of any patient with van Buchem's disease undergoing surgical decompression for this. It is of note that both van Buchem's original patients and a more recent patient were mentally subnormal ${ }^{14}$ and it may be that intellectual function was preserved in the present patients by early surgical relief of the raised intracranial pressure. Both patients reported here recovered from their early symptoms after surgery only to develop problems in later life. The first patient was disabled due to cerebellar compression by encroachment on the posterior cranial fossa and our second patient developed spastic paraparesis due to compression of the dorsal spinal cord by the grossly involved vertebral column. Such problems are not uncommon and blindness and epilepsy have also been reported. ${ }^{24}$

In most reported series, ${ }^{25}$ van Buchem's disease appears to be transmitted as an autosomal recessive trait. In the two cases reported here, there is no evidence of the disease in either parent, nor in any of the generation subsequent to the patients themselves. All the cases in this family appear in the same sibship, but it is remarkable that four out of six sibs were affected. The disease is probably transmitted as autosomal recessive in this family, despite this unexpected frequency. In several series however, the disease has been thought to be transmitted as an autosomal dominant ${ }^{368}$ but in these, the radiological changes have been less marked and the patients often asymptomatic.

The clinical course of these two patients with van Buchem's disease indicates the relatively good prognosis, even when presenting with raised intracranial pressure, if the appropriate decompressive surgery is carried out in early adult life. Failure to do this may result in blindness and even dementia in some patients.

We thank Dr C Mawdsley and Mr P Edmond for their permission to report patients under their care, and Miss C Hughes for secretarial assistance.

\section{References}

I Van Buchem FSP, Hadders HN, Ubbens R. An uncommon familial systemic disease of the skeleton. Hyperostosis corticalis generalisata familiaris. Acta Radiol 1955;44:109-19.

2 Van Buchem FSP, Hadders HN, Hansen JF, Woldring MG. Hyperostosis corticalis generalisata. Report of seven cases. Am J Med 1962;33:387-97.

3 Worth HM, Wollin DG. Hyperostosis corticalis generalisata congenita. J Can Assoc Radiol 1966; 17:67-74.

4 Fosmoe RJ, Holm RS, Roscoe CM. Van Buchem's disease (hyperostosis corticalis generalisata familiaris). A case report. Radiology 1968;90:771-4.

5 Van Buchem FSP. Hyperostosis corticalis generalisataeight new cases. Acta Med Scand 1971;189:257-67.

- Maroteaux P, Fontaine G, Scharfman W, Farriaux JP. L'hypertostose corticale generalisee, a transmission dominante. Arch Fr pediat 1971;28:685-98. 
7 Scott WC, Gautby THT. Hyperostosis corticalis generalisata familiaris. Br J Radiol 1974;47:500-3.

8 Owen RH. Van Buchem's disease (hyperostosis corticalis generalisata). Br J Radiol 1976;49:126-32.

9 Jacobs P. Van Buchem's disease. Postgrad Med J 1977;53:597-605.
${ }^{10}$ Higenbotham NL, Alexander SK. Osteopetrosis, four cases in one family. Am J Surg 1941;53:444-54.

11 Johnston CC, Lavy N, Lord T, Vellios F, Merritt AD, Deiss WP. Osteopetrosis. Medicine 1968;47,3:144-67.

12 Hinkell CL, Beiler DD. Osteopetrosis in adults. Am J Roentgenol 1955;74:46-64. 